\title{
Conics on a Maschke Surface
}

By W. L. Edge.

(Received 24th April, 1945. Read 2nd June, 1945.)

1. The six quaternary quartic forms

$$
\begin{aligned}
& \Phi_{1} \equiv x^{4}+y^{4}+z^{4}+t^{4}-6\left(y^{2} z^{2}+z^{2} x^{2}+x^{2} y^{2}+x^{2} t^{2}+y^{2} t^{2}+z^{2} t^{2}\right), \\
& \Phi_{2} \equiv x^{4}+y^{4}+z^{4}+t^{4}+6\left(-y^{2} z^{2}+z^{2} x^{2}+x^{2} y^{2}-x^{2} t^{2}+y^{2} t^{2}+z^{2} t^{2}\right), \\
& \Phi_{3} \equiv x^{4}+y^{4}+z^{4}+t^{4}+6\left(y^{2} z^{2}-z^{2} x^{2}+x^{2} y^{2}+x^{2} t^{2}-y^{2} t^{2}+z^{2} t^{2}\right), \\
& \Phi_{4} \equiv x^{4}+y^{4}+z^{4}+t^{4}+6\left(y^{2} z^{2}+z^{2} x^{2}-x^{2} y^{2}+x^{2} t^{2}+y^{2} t^{2}-z^{2} t^{2}\right), \\
& \Phi_{5} \equiv-2\left(x^{4}+y^{4}+z^{4}+t^{4}\right)-24 x y z t, \\
& \Phi_{6} \equiv-2\left(x^{4}+y^{4}+z^{4}+t^{4}\right)+24 x y z t,
\end{aligned}
$$

were first obtained by Maschke; ${ }^{1}$ it has recently been explained ${ }^{2}$ that the quartic surfaces obtained by equating these forms to zero are important constituents of Klein's famous configuration derived from six linear complexes that are mutually in involution. The quartic surface $\Phi_{i}=0$ will be denoted, for each of the six suffixes $i$, by $M_{i}$.

Each of the differences $\Phi_{i}-\Phi_{j}$ has four linear factors, so that the pencil determined by any two of the six Maschke surfaces includes a tetrahedron; the fifteen fundamental tetrahedra of Klein are all accounted for in this way. The tetrahedron which belongs to the pencil determined by $M_{i}$ and $M_{j}$ will be denoted ${ }^{3}$ by $U_{i j}$, the order of the two suffixes being immaterial. Three tetrahedra such as $U_{i j}, U_{j k}$, $U_{k i}$, whose pairs of suffixes are formed from only three of the six digits, constitute a desmic triad. There are 16 lines containing vertices of each of the three tetrahedra and another 16 lines lying in faces of each of the three tetrahedra. If $l, m, n$ are the three digits other than $i, j, k$ these same 32 lines arise from the desmic triad $U_{m n}$, $U_{n l}, U_{l m}$; but a line containing vertices of the former triad lies in faces of the latter while a line lying in faces of the former triad contains vertices of the latter. These 32 lines are $f$-lines $(E, \S 2)$; the six digits are separable into complementary triads in ten different

3 Math. Annalen, 17 (1880), 510-516.

2 Proc. Edin. Math. Soc. (2), 7 (1945), 93-103. This paper will be alluded to as $E$.

3 The notation for the tetrahedra which was used in $E$ is brought into line by replacing the suffix 0 by 5 and $T_{i}$ by $U_{i 6}$. 
ways, and the whole set of $320 \mathrm{f}$-lines mentioned by Klein is thus accounted for.

There are five of the fundamental tetrahedra, namely those with $i$ for one of their suffixes, which belong to pencils determined by $M_{i}$ and another Maschke surface: these tetrahedra will now be described as syzygetic to $M_{i}$, while the remaining ten tetrahedra, among whose suffixes $i$ does not appear, will be said to be azygetic to $M_{i}$. Now any tetrahedron gives rise to an abelian group of eight collineations; these consist, in addition to the identical operation, of four harmonic inversions each involving a vertex and the opposite face and of three harmonic inversions each involving a pair of opposite edges. If a surface should be invariant for all these operations, then it may be said to be its own harmonic inverse in the tetrahedron; a Maschke surface is its own harmonic inverse in any azygetic tetrahedron. Suppose then that $O$ is any vertex and $\omega$ the opposite face of a tetrahedron azygetic to a Maschke surface $M$. Then, if $P$ is any point of $M$, the harmonic conjugate $P^{\prime}$ of $P$ with respect to $O$ and the intersection of $O P$ with $\omega$ is also a point of $M$. If, in particular, $P$ is any point of the section of $M$ by $\omega$ then $P^{\prime}$ coincides with $P$; thus every line which joins $O$ to a point of the section of $M$ by $\omega$ is a tangent of $M$ at this latter point. The cubic surface which is the first polar of $O$ with respect to $M$ must therefore contain the plane quartic curve in which $\omega$ meets $M$, and so must contain the whole of the plane $\omega$. This polar therefore consists of $\omega$ and a quadric.

\section{Pairs of. conics with four distinct intersections.}

2. Any pair of opposite edges of a fundamental tetrahedron belongs also to two other fundamental tetrahedra. There are 15 of these pairs of directrices, and the 15 triads of tetrahedra which have pairs of directrices in common correspond to the synthemes of Sylvester, that is to the divisions of six objects into three pairs. For example: $U_{i j}, U_{k l}, U_{m n}$ have a pair of directrices in common. It follows that when any one of the six Maschke surfaces and any one of the fifteen pairs of directrices are given the three tetrahedra to which these directrices belong are two of them azygetic and one of them syzygetic to the surface.

Suppose then that $M$ is a Maschke surface, $d, e$, a pair of directrices, $U, U^{\prime}$ those tetrahedra to which $d$ and $e$ belong and which are azygetic to $M$. Let $O_{1}, O_{2}$ be the vertices of $U$ on $d$, and $O_{3}, O_{4}$ the vertices of $U$ on $e ; \omega_{1}, \omega_{2}, \omega_{3}, \omega_{4}$ the faces of $U$ opposite $O_{1}, O_{2}$, 
$O_{3}, O_{4}$ respectively. Similarly let $O_{1}^{\prime}, O_{2}^{\prime}$ be the vertices of $U^{\prime}$ on $d$, and $O_{3}^{\prime}, O_{4}^{\prime}$ the vertices of $U^{\prime}$ on $e ; \omega_{1}^{\prime}, \omega_{2}^{\prime}, \omega_{3}^{\prime}, \omega_{4}^{\prime}$ the faces of $U^{\prime}$ opposite $O_{1}^{\prime}, O_{2}^{\prime}, O_{3}^{\prime}, O_{4}^{\prime}$ respectively. We consider the tangent planes. of $M$ which pass through $d$; their points of contact are the intersections of $M$ with the base curve $C$ of the pencil of first polars of the points of $d$. Now $e$, which is common to the planes $\omega_{1}, \omega_{2}$ forming parts of the respective polars of $O_{1}, O_{2}$, belongs to $C$; the rest of $C$ consists of four conics, one in each of $\omega_{1}, \omega_{2}, \omega_{1}^{\prime}, \omega_{2}^{\prime}$. For $\omega_{1}$ is part of the polar of $O_{1}$, so that the conic $\gamma_{1}$ in which it is met by the quadric belonging to the polar of $O_{2}$ belongs to $C$; incidentally the quadrics which belong to the polars of $O_{2}, O_{1}^{\prime}, O_{2}^{\prime}$ must all meet $\omega_{1}$ in this same conic $\gamma_{1}$. Similarly there are conics $\gamma_{2}, \gamma_{1}^{\prime}, \gamma_{2}^{\prime}$ in $\omega_{2}, \omega_{1}^{\prime}, \omega_{2}^{\prime}$; the whole of $C$, whose order is 9 , is thus accounted for.

$M$ is its own harmonic inverse in both $U$ and $U^{\prime}$, and from this it follows that the intersections of $\gamma_{1}, \gamma_{2}, \gamma_{1}^{\prime}, \gamma_{2}^{\prime}$ with $M$ lie four in each of eight planes through $d$, which planes are therefore quadritangent planes of $M$. For suppose that $P$ is a point common to $M$ and $\gamma_{1}$, so lying in $\omega_{1}$ and being a point of contact with $M$ of a tangent plane through $d$; then all points derived from $P$ by harmonic inversions in $U$ or $U^{\prime}$ must also be points of contact with $M$ of tangent planes through $d$, and some of these inversions, though altering the position of $P$, leave the plane $d P$ unaffected. For example: inversion by means of $O_{1}^{\prime}$ and $\omega_{1}^{\prime}$ changes $P$ to a point of $\omega_{2}$ while inversion by means of $O_{2}^{\prime}$ and $\omega_{2}^{\prime}$ changes $P$ to a second point of. $\omega_{2}$, for it will be remembered that $\omega_{1}$ and $\omega_{2}$ are harmonically conjugate to $\omega_{1}^{\prime}$ and $\omega_{2}^{\prime}$. Neither of these inversions alters the plane $d P$. Their product is the biaxial harmonic inversion with axes $d$ and $e$; this inversion, while interchanging the two points of $\omega_{2}$ already derived from $P$, changes $P$ to another point of $\omega_{1}$. There are thus, including $P$, four points at each of which the plane $d P$ must touch $M$; two of the points are on $\gamma_{1}$ and two on $\gamma_{2}$, the quadrangle which they form having $O_{1}^{\prime}$ and $O_{2}^{\prime}$ for two of its diagonal points while the third diagonal point is on $e$. There are thus eight planes through $d$, each of which touches $M$ in four distinct points; the four points are either two on each of $\gamma_{1}, \gamma_{2}$ or two on each of $\gamma_{1}^{\prime}, \gamma_{2}^{\prime}$. The intersection of such a plane with $M$ is a quartic curve with nodes at the four points and which is invariant for the three harmonic inversions arising from their diagonal triangle: it is thus, presumably, a pair of conics of which the four points are the intersections. The algebra below shows that this is so: it also shows that the four pairs of conics in which $M$ is met by those of the quadritangent planes whose contacts are on $\gamma_{1}$ and $\gamma_{2}$ make up the 
intersection of $M$ with a pair of -quadrics, and there is a corresponding result for the other four planes.

The six Maschke surfaces are, so far as $d$ is concerned, paired; for $d$ belongs to three fundamental tetrahedra and each of these is syzygetic to two of the surfaces. The quadritangent planes through $d$ are the same for the two surfaces of any pair. This follows because the harmonic inversions involving either of the two vertices of the syzygetic tetrahedron on $d$ interchange the pair of surfaces while leaving the quadritangent planes unchanged (though the points of contact in the planes will be altered).

3. Let us obtain equations for the quadritangent planes to the Maschke surfaces through the directrix $z=t=0$. Since

$$
\Phi_{4}-\Phi_{1} \equiv 12\left(x^{2}+y^{2}\right)\left(z^{2}+t^{2}\right), \quad \Phi_{2}-\Phi_{3} \equiv 12\left(x^{2}-y^{2}\right)\left(z^{2}-t^{2}\right),
$$

and

$$
\Phi_{6}-\Phi_{5} \equiv 48 x y z l
$$

the pair of directriees $z=t=0$ and $x=y=0$ belongs to $U_{14}, U_{23}$ and $U_{56}$.

Consider $M_{5}$. The two azygetic tetrahedra are $U_{14}$, with vertices $(1, i, 0,0)$ and $(1,-i, 0,0)$ on $z=t=0$ and $U_{23}$, with vertices $(1,1,0,0)$ and $(1,-1,0,0)$ on $z=t=0$. The base curve of the first polars of the points of $z=t=0$ is found to consist, apart from the line $x=y=0$, of the four conics

$$
\begin{array}{ll}
x+y=y^{2}-3 z t=0, & x+i y=y^{2}-3 i z t=0, \\
x-y=y^{2}+3 z t=0, & x-i y=y^{2}+3 i z t=0 .
\end{array}
$$

What of the intersections of these conics with $M_{5}$ ? The equations

$$
\Phi_{5}=x+y=y^{2}-3 z t=0
$$

give, on eliminating $x$ and $y, z^{4}-18 z^{2} t^{2}+t^{4}=0$, as also do the equations

$$
\Phi_{5}=x-y=y^{2}+3 z t=0 .
$$

Thus four of the quadritangent planes of $M_{5}$ which pass through $z=t=0$ are found. The other four, obtained by elimination of $x$ and $y$ from either of the sets of equations

$$
\begin{aligned}
& \Phi_{\overline{5}}=x+i y=y^{2}-3 i z t=0, \\
& \Phi_{\overline{5}}=x-i y=y^{2}+3 i z t=0,
\end{aligned}
$$

are given by $z^{4}+18 z^{2} t^{2}+t^{4}=0$.

It is natural to consider for a moment the pencil of quartic surfaces determined by $M_{5}$ and one of these two sets of four quadritangent planes. One of the two pencils is given by 


$$
\lambda\left(z^{4}+18 z^{2} t^{2}+t^{4}\right)-2^{*}\left(x^{4}+y^{4}+z^{4}+t^{4}+12 x y z t\right)=0 .
$$

If $\lambda=2$ the terms in $z^{4}$ and $t^{4}$ disappear, and the resulting surface consists of two quadrics; for the left-hand side of the equation then becomes $36 z^{2} t^{2}-2\left(x^{4}+y^{4}+12 x y z t\right)$, which is the same as $(6 z t-2 x y)^{2}-2\left(x^{2}+y^{2}\right)^{2}$. Thus

$$
2\left(z^{4}+18 z^{2} t^{2}+t^{4}\right)+\Phi_{5} \equiv 4(3 z t-x y)^{2}-2\left(x^{2}+y^{2}\right)^{2} .
$$

Similarly

$$
2\left(z^{4}-18 z^{2} t^{2}+t^{4}\right)+\Phi_{5} \equiv-4(3 z t+x y)^{2}-2\left(x^{2}-y^{2}\right)^{2} .
$$

The curve in which any one of the eight quadritangent planes through $z=t=0$ meets $M_{5}$ is therefore the same as the curve in which it meets the pair of quadrics obtained by factorising the right-hand side of one of the above identities, and so consists of two conics.

Two corresponding identities involving $\Phi_{6}$ are obtained immediately by changing the sign of any one of $x, y, z, t$; so far as the directrix $z=t=0$ is concerned, $M_{5}$ and $M_{6}$ have the same quadritangent planes.

Identities involving the remaining four forms are obtainable in a similar way; Consider the quadritangent planes of, for example, $M_{1}$ which pass through $z=t=0$. The two azygetic tetrahedra are now $U_{23}$ and $U_{56}$; the four conics which belong to the base curve of the pencil of first polars are found to be

$$
\begin{aligned}
& x=y^{2}-3\left(z^{2}+t^{2}\right)=0, \quad x+y=2 y^{2}+3\left(z^{2}+t^{2}\right)=0, \\
& y=x^{2}-3\left(z^{2}+t^{2}\right)=0, \quad x-y=2 y^{2}+3\left(z^{2}+t^{2}\right)=0 .
\end{aligned}
$$

The elimination of $x$ and $y$ between either of the sets of equations

$$
\begin{aligned}
& \Phi_{1}=x=y^{2}-3\left(z^{2}+t^{2}\right)=0, \\
& \Phi_{1}=y=x^{2}-3\left(z^{2}+t^{2}\right)=0,
\end{aligned}
$$

gives $z^{4}+3 z^{2} t^{2}+t^{4}=0$, while elimination between either of the sets of equations

$$
\begin{aligned}
& \Phi_{1}=x+y=2 y^{2}+3\left(z^{2}+t^{2}\right)=0, \\
& \Phi_{1}=x-y=2 y^{2}+3\left(z^{2}+t^{2}\right)=0,
\end{aligned}
$$

gives $5 z^{4}+6 z^{2} t^{2}+5 t^{4}=0$. And we obtain the identities

$$
\begin{aligned}
& 8\left(z^{4}+3 z^{2} t^{2}+t^{4}\right)+\Phi_{1} \equiv\left\{x^{2}+y^{2}-3\left(z^{2}+t^{2}\right)\right\}^{2}-8 x^{2} y^{2}, \\
& 2\left(5 z^{4}+6 z^{2} t^{2}+5 t^{4}\right)-\Phi_{1} \equiv\left\{x^{2}+y^{2}+3\left(z^{2}+t^{2}\right)\right\}^{2}-2\left(x^{2}-y^{2}\right)^{2} .
\end{aligned}
$$

Identities involving $\Phi_{4}$ are found from these by writing $i x$ for $x$ and $i y$ for $y$, for this changes $\Phi_{1}$ into $\Phi_{4}$; the sets of quadritangent planes through $z=t=0$ are not altered thereby. If, on the other hand, in 
the identities involving $\Phi_{1}, y$ is replaced by iy and $z$ by $i z$, two identities involving $\Phi_{2}$ appear while, if $z$ is replaced by $i z$ and $x$ by $i x$, identities involving $\Phi_{3}$ are obtained.

There are then eight quadritangent planes of each of the six Maschke surfaces passing through each of the 30 directrices. Any Maschke surface has therefore 240 plane sections consisting of pairs of conics with four distinct intersections.

\section{Pairs of conics with double contact.}

4. The sum of any three of the six forms $\Phi$ is a perfect square; thus the 64 intersections of any three of the Maschke surfaces consist of only 32 points at each of which the three surfaces have a common tangent line, and the 32 points lie on a quadric. Since $\Sigma \Phi_{i}$ vanishes identically the repeated quadric $\Phi_{i}+\Phi_{j}+\Phi_{k}=0$ is the same as $\Phi_{l}+\Phi_{m}+\Phi_{n}=0$; these ten quadrics are Klein's fundamental quadrics, which are thus all accounted for. Now the tetrahedra $U_{i j}$, $U_{j k}, U_{k i}$ of a desmic triad belong to a pencil of quartic surfaces whose base curve consists of the $16 f$-lines common to the faces of the tetrahedra; since, for any $i$ and $j$, the pencil of quartic surfaces determined by $M_{i}$ and $M_{j}$ includes $U_{i j}$, the 32 points for which $\Phi_{i}=\Phi_{j}=\Phi_{k}=0$ must lie two on each of these $16 f$-lines, which must be bitangents of all three Maschke surfaces. There arise in this way $160 f$-lines bitangent to any Maschke surface; they lie four in each of the faces of the ten azygetic tetrahedra.

The points commion to three, of the set of six, Maschke surfaces are parabolic points on all of them. For consider any point for which three of the six forms $\Phi$ simultaneously vanish. At such a point, in virtue of Maschke's identity $4 \Sigma \Phi_{i} \Phi_{j} \Phi_{k} \Phi_{l} \equiv\left\{\Sigma \Phi_{i} \Phi_{j}\right\}^{2}, \Sigma \Phi_{i} \Phi_{j}$ must vanish and therefore, since $\Sigma \Phi_{i}^{2} \equiv\left(\Sigma \Phi_{i}\right)^{2}-2 \Sigma \Phi_{i} \Phi_{j}, \Sigma \Phi_{i}^{2}$ vanishes too. The point is therefore $(E, \S 6)$ parabolic on any of the six Maschke surfaces on which it lies.

Let $\lambda$ be any one of the $160 f$-lines bitangent to a Maschke surface $M$; of the three tetrahedra one of whose faces contains $\lambda$ there is one, $U$ say, azygetic to $M$; let $O$ be the vertex of $U$ opposite to that face $\omega$ which contains $\lambda$. Since the line joining $O$ to any point of the section of $M$ by $\omega$ touches $M$ at this latter point the tangent plane of $M$ at either of its points of contact $I, J$, with $\lambda$ joins $\lambda$ to $O$; the same tangent plane arises for both contacts. But, since $I$ and $J$ are parabolic points of $M$, the section of $M$ by the plane $O \lambda$ must have cusps at $I$ and $J$; moreover, since $U$ is azygetic to $M$, the cuspidal 
tangents of the curve of section at $I$ and $J$ must remain invariant for harmonic inversion by means of $O$ and $\omega$. The cuspidal tangent at $I$ must then be either $O I$ or $I J$; it cannot be $I J$ because $\lambda$ has only two-point contact with $M$ at $I$, so that it is $O I$. Similarly $O J$ is the cuspidal tangent at $J$. Further: any line through $O$ which has threepoint contact with $M$ at a point of its section by $\omega$ must, since $M$ is invariant for the harmonic inversion, have four-point contact there; it follows that the cusps at $I$ and $J$ of the section by the plane $O \lambda$ are not ordinary cusps but tacnodes. The quartic curve of section; having tacnodes at both $I$ and $J$, must break up: the presumption that it is a pair of conics having double contact at $I$ and $J$ is vindicated by the algebra below. There are therefore 160 planes which meet a Maschke surface in pairs of conics having double contact.

It is seen incidentally that $I$ and $J$ are not only on the parabolic curve but also on the flecnodal curve of $M$; this curve, so named by Salmon, is the locus of points of $M$ at which there is a tangent line having four-point contact. Thus $I$ and $J$ are points of contact of the parabolic and flecnodal curves which, as shown by Clebsch, ${ }^{1}$ touch wherever they meet. Clebsch also gave the number of these contacts which, for a quartic surface, is 320 ; thus all these points on $M$ are accounted for by its contacts with the 160 bitangent $f$-lines.

5. The tetrahedron of reference $U_{56}$ is azygetic to $M_{1}$; those $f$-lines which lie in a face of $U_{56}$ and are bitangent to $M_{1}$ are the intersections of this face with the faces

$-x+y+z+t=0, \quad x-y+z+t=0, \quad x+y-z+t=0, \quad x+y+z-t=0$, of $U_{16}$, and the corresponding tangent planes of $M_{1}$ join these lines to the opposite vertex of $U_{56}$. Thus the equations of the tangent planes of $M_{1}$ at its points of contact with its bitangent $f$-lines which lie in the plane $x=0$ are

$$
y+z+t=0, \quad z+t-y=0 ; \quad t+y-z=0, \quad y+z-t=0 .
$$

The curves of intersection of these four planes with $M_{1}$ together constitute the base of the pencil of quartic surfaces

$$
\begin{gathered}
\Phi_{1}+\kappa\left(y^{4}+z^{4}+t^{4}-2 z^{2} t^{2}-2 t^{2} y^{2}-2 y^{2} z^{2}\right)=0 \\
\left\{x^{2}-3\left(y^{2}+z^{2}+t^{2}\right)\right\}^{2}=(8-\kappa)\left(y^{4}+z^{4}+i^{4}\right)+2(\kappa+12)\left(z^{2} t^{2}+t^{2} y^{2}+y^{2} z^{2}\right) .
\end{gathered}
$$

If, now, $\kappa$ is chosen so that $\kappa+12=8-\kappa$, the right-hand side is a

1. Journal fïr Math., 58 (1861), 93-108 (105). 
square and the resulting surface of the pencil a pair of quadrics. And we have the identity

$$
\begin{aligned}
\Phi_{1} & -2(y+z+t)(z+t-y)(t+y-z)(y+z-t) \\
& \equiv\left\{x^{2}-3\left(y^{2}+z^{2}+t^{2}\right)\right\}^{2}-6\left(y^{2}+z^{2}+t^{2}\right)^{2} \\
& \equiv 3\left(y^{2}+z^{2}+t^{2}-x^{2}\right)^{2}-2 x^{4}
\end{aligned}
$$

This identity shows that a plane obtained by equating to zero a factor of the second term on the left meets $M_{1}$ in the same curve as that in which it meets the pair of quadrics obtained by equating to zero the right-hand side. Since these quadrics have ring-contact along the conic $x=y^{2}+z^{2}+t^{2}=0$, each of the four planes meets $M_{1}$ in a pair of conics having double contact.

There is a corresponding identity for any one of the Maschke surfaces and any set of bitangent $f$-lines in a face of an azygetic tetrahedron; these identities can be obtained either directly or else by imposing suitable linear substitutions on the variables and using the above identity. Perhaps a few lines should be devoted to one of the surfaces $M_{5}$ or $M_{6}$.

Let us take those four bitangent lines of $M_{6}$ in which the faces $x=0, y=0, z=0, t=0$ of $U_{56}$ meet the face $x+y+z+t=0$ of the azygetic tetrahedron $U_{15}$; they are joined to the opposite vertex $(1,1,1,1)$ of $U_{15}$ by the planes

$$
y+z+t-3 x=0, z+t+x-3 y=0, t+x+y-3 z=0, x+y+z-3 t=0 .
$$

This points to the consideration of the pencil of surfaces

$$
\mu \Phi_{6}+(y+z+t-3 x)(z+t+x-3 y)(t+x+y-3 z)(x+y+z-3 t)=0,
$$

and the above discussion concerning $M_{1}$ leads us to expect that there is a value of $\mu$ for which the resulting surface of the pencil breaks up into a pair of quadrics. And it happens, when $\mu=-32$, that

$$
\begin{array}{r}
16 \begin{array}{r}
\left(x^{4}+y^{4}+z^{4}+t^{4}-12 x y z t\right)+(y+z+t-3 x)(z+t+x-3 y) \\
(t+x+y-3 z)(x+y+z-3 t)
\end{array} \\
\equiv 2\left\{3\left(x^{2}+y^{2}+z^{2}+t^{2}\right)+2(y z+z x+x y+x t+y t+z t)\right\}^{2}-5(x+y+z+t)^{4} .
\end{array}
$$

Conics on the Hessian of a Maschke surface.

6. It has been remarked that if $O$ is a vertex and $\omega$ the opposite face of a tetrahedron azygetic to a Maschke surface $M$, the first polar of $O$ consists of the plane $\omega$ and a quadric. Hence, since any point of the conic $\gamma$ common to $\omega$ and the quadric is a node on the first polar of $O$, the polar quadric of the point has a node at $O ; \gamma$ there- 
fore lies on the Hessian $H$ of $M . \quad H$ thus contains 40 conics, one in each face of each azygetic tetrahedron. These conics actually make up the complete intersection of the Hessian with the fundamental quadrics of Klein.

The Hessian of $\Phi_{1}$ may be taken as

$$
-\left|\begin{array}{cccc}
x^{2}-y^{2}-z^{2}-t^{2} & -2 x y & -2 x z & -2 x t \\
-2 x y & y^{2}-z^{2}-t^{2}-x^{2} & -2 y z & -2 y t \\
-2 x z & -2 y z & z^{2}-t^{2}-x^{2}-y^{2} & -2 z t \\
-2 x t & -2 y t & -2 z t & t^{2}-x^{2}-y^{2}-z^{2}
\end{array}\right|,
$$

or

$$
\begin{aligned}
\left(x^{2}+y^{2}+z^{2}+t^{2}\right)^{4}-32\left(x^{2}+y^{2}+z^{2}+t^{2}\right)\left(y^{2} z^{2} t^{2}+z^{2} t^{2} x^{2}+t^{2} x^{2} y^{2}+x^{2} y^{2} z^{2}\right) \\
+256 x^{2} y^{2} z^{2} t^{2} \\
+
\end{aligned}
$$

This, when equated to zero, gives the Hessian surface $H_{1}$ of $M_{1}$, and the form shows that the four conics of $H_{1}$ which lie in the faces of the azygetic tetrahedron $U_{56}$ all lie on the fundamental quadric $x^{2}+y^{2}+z^{2}+t^{2}=0$ and, moreover, that the complete intersection of this quadric with $H_{1}$ consists of these four conics, counted twice.

Any synthematic total of tetrahedra syzygetic to a Maschke surface is such that two of them are self-polar and the other three inscribed to any one of the fundamental quadrics; the ten fundamental quadrics correspond in this way to the pairs of self-polar tetrahedra that they respectively possess in the synthematic total. The azygetic tetrahedron that forms a desmic triad with the pair of self-polar tetrahedra is the one whose four plane sections of the fundamental quadric lie on the Hessian of the Maschke surface.

Mathematical Institute, The University, Edinborgh. 